\title{
Genetic basis of the very short life cycle of 'Apogee' wheat
}

\author{
Genqiao Li ${ }^{1,3 \dagger}$, Rungravee Boontung ${ }^{2 \dagger}$, Carol Powers ${ }^{1 \dagger}$, Vikas Belamkar ${ }^{2}$, Tianrong Huang ${ }^{1,4}$, Fang Miao ${ }^{1,5}$,
}

P. Stephen Baenziger ${ }^{2^{*}}$ and Liuling Yan ${ }^{* *}$

\begin{abstract}
Background: 'Apogee' has a very short life cycle among wheat cultivars (flowering 25 days after planting under a long day and without vernalization), and it is a unique genetic material that can be used to accelerate cycling breeding lines. However, little is known about the genetic basis of the super-short life of Apogee wheat.

Results: In this study, Apogee was crossed with a strong winter wheat cultivar 'Overland', and $858 \mathrm{~F}_{2}$ plants were generated and tested in a greenhouse under constant warm temperature and long days. Apogee wheat was found to have the early alleles for four flowering time genes, which were ranked in the order of $v r n-A 1>V R N-B 1>v r n-$ D3 > PPD-D1 according to their effect intensity. All these Apogee alleles for early flowering showed complete or partial dominance effects in the $F_{2}$ population. Surprisingly, Apogee was found to have the same alleles at vrn-A1a and vrn-D3a for early flowering as observed in winter wheat cultivar 'Jagger.' It was also found that the vrn-A1a gene was epistatic to VRN-B1 and vrn-D3. The dominant vrn-D3a alone was not sufficient to cause the transition from vegetative to reproductive development in winter plants without vernalization but was able to accelerate flowering in those plants that carry the vrn-A1a or Vrn-B1 alleles. The genetic effects of the vernalization and photoperiod genes were validated in Apogee $\times$ Overland $F_{3}$ populations.

Conclusion: VRN-A1, VRN-B1,VRN-D3, and PPD-D1 are the major genes that enabled Apogee to produce the very short life cycle. This study greatly advanced the molecular understanding of the multiple flowering genes under different genetic backgrounds and provided useful molecular tools that can be used to accelerate winter wheat breeding schemes.
\end{abstract}

Keywords: Apogee wheat, Flowering genes, Vernalization, Photoperiod, VRN1

\section{Background}

Two wheat (Triticum aestivum L. $2 \mathrm{n}=6 \mathrm{x}=42$, AABBDD) cultivars, 'USU-Apogee' and 'USU-Perigee', were developed and released in 1996 by the Utah Agricultural Experiment Station at Utah State University (USU) in cooperation with the National Aeronautics and Space Administration (NASA) for improved food crops for the Advanced Life Support System (ALSS) in a space station [1]. Apogee is named for the point in an orbit farthest from the earth, while Perigee is named for the point in an

\footnotetext{
* Correspondence: pbaenziger1@unl.edu; liuling.yan@okstate.edu

${ }^{\dagger}$ Equal contributors

2Department of Agronomy and Horticulture, University of Nebraska-Lincoln, Lincoln, NE 68583, USA

'Department of Plant and Soil Sciences, Oklahoma State University, Stillwater OK74078, USA

Full list of author information is available at the end of the article
}

orbit closest to the earth. The two wheat cultivars were used to regenerate oxygen, purify water, and produce food [2]. Due to restricted space, one of the major requirements for a wheat cultivar adapted to grow in the ALSS food system is a short cycle life.

Apogee can flower 25 days after planting when grown under constant conditions $\left(23{ }^{\circ} \mathrm{C}\right.$ warm temperature and continuous light) [3], which is the shortest life cycle in wheat in the world. Under the same conditions, Perigee flowers 30 days after planting and also displays an extremely rapid development rate. In addition to the unique rapid life cycle, the two hard red spring wheat cultivars have several other properties suitable for the space station, including dwarf stature that allow the plants to grow on shelves, resistance to the calcium-induced leaf tip necrosis that occurs in rapid growth conditions, and high grain yield, particularly in high temperature environments $[2,4,5]$. 
Therefore, Apogee and Perigee are elite genetic materials for breeding rapid cycling lines.

The exceptionally short life of Apogee and Perigee is mainly due to their spring growth habit. Unlike winter wheat varieties that require an extended exposure to low temperatures to accelerate flowering (vernalization, VRN), Apogee and Perigee flower under temperature-photoperiod controlled conditions and without vernalization. The developmental process of wheat is mainly controlled by genes in three pathways, including vernalization response, photoperiod response (PPD), and earliness per se (EPS) [5-7]. Among thestrongest effective mechanism underlying wheat flowering is vernalization that distinguishes spring wheat and winter wheat $[8,9]$. Major genes responsible for the difference in vernalization requirement between the two distinct types have been cloned, including VRN-1 [10], VRN-3 [11], and $V R N-D 4$ [12] whose expression is promoted by low temperature, and VRN-2 [13] that is repressed by low temperature. The wheat PPD genes for photoperiod sensitivity were isolated [14] based on77 the sequence of the orthologous PPD-H1 gene in barley [15]. No EPS gene for earliness per se has been cloned from either spring or winter wheat cultivars $[7,16]$.

Apogee and Perigee are cultivars of common hexaploid wheat that has three homoeologous genomes $\mathrm{A}, \mathrm{B}$, and $\mathrm{D}$, forming a series of homoeologous genes, such as VRN-A1, $V R N-B 1$, and $V R N-D 1$ on chromosomes $5 \mathrm{AL}, 5 \mathrm{BL}$, and 5DL [17]. The homoeologous genes have high identity in sequence in genetic backgrounds in different wheat cultivars, making it complicated to genotype a specific cultivar for three homoeologous genes. The existence of multiple alleles in a wheat homoeologous gene could even make it more complicated to genotype a specific cultivar. VRN1 was first cloned from diploid wheat $T$. monococcum $\left(2 n=2 \times=14\right.$, genome $\left.\mathrm{A}^{\mathrm{m}} \mathrm{A}^{\mathrm{m}}\right)$ [10], and five spring alleles of $V r n-A^{m} 1$, varying in deletion lengths (alleles $V r n-A^{m} 1 a$, $\left.V r n-A^{m} 1 b, V r n-A^{m} 1 g, V r n-A^{m} 1 f\right)$, are involved in a socalled CArG-box recognition site [18-20], compared with the recessive $v r n-A^{m} 1$ allele for winter wheat. A recent study reports a novel allele, $V r n-A 1 u$,that has a small deletion in the promoter region in diploid $T$. urartu $(2 n=2 \times=14$, genome AA) [21]. Two alleles that have different deletions in the promoter (Vrn-Ald and Vrn-Ale) and one allele that has a large deletion in intron one ( $\mathrm{Vrn}$ A1c) were observed in tetraploid wheat [18]. In hexaploid spring wheat, some cultivars have a deletion in the promoter $(V r n-A 1 b)$, but more than half of varieties have the $V r n-A 1 a$ allele that has a miniature inverted-repeat transposable element (MITE) inserted in its promoter ( $\mathrm{Vrn}$ A1a) (MITE_VRN) [18]. The MITE_VRN can be recognized by microRNA TamiRNA1123 [22]. While the regulatory elements are found in the promoter or intron one in spring wheat, single nucleotide polymorphisms (SNPs) found in coding regions explain quantitative variation in vernalization requirements in winter wheat. While the $v r n-A 1 a$ allele in weak winter wheat 'Jagger' requires less cumulative low temperatures and the $v r n-A 1 b$ allele in the stronger winter wheat '2174' requires more cumulative low temperatures to reach a vernalization saturation point, the two vrn-A1 alleles rely on variation not in the promoter or intron but at the protein level [23].

Compared with the many $V R N-A 1$ alleles as reviewed above, $V R N-B 1$ and $V R N-D 1$ have fewer observed haplotypes. Vrn-B1 in spring wheat has a deletion in intron one in hexaploid wheat and tetraploid T. turgidum ssp. durum $[10,18,24]$ or a $5.6 \mathrm{~kb}$ retrotransposable element (Retrotrans_VRN) in the 5'-untranslated region (UTR) in tetraploid T. turgidum subsp. carthlicum [25]. Vrn-D1 in spring wheat has a large deletion in intron one in hexaploid wheat [24].

Relative to the multiple alleles in the VRN1 genes, other genes known to regulate flowering time have fewer haplotypes. In spring wheat cultivars, the Vrn-B3 allele has the insertion of a retroelement in its promoter region in the cultivar Hope [11], and Vrn-A3 and Vrn-D3 have variation in the non-coding intronic region [26]. In winter wheat cultivars, allelic variation between vrn-D3a for early flowering and $v r n-D 3 b$ for late flowering is due to a $(G)_{3 o r 4}$ polymorphism in exon 3 that results in a frame shift involving 81 amino acids [26-28]. In both spring wheat and winter wheat, allelic variation between $P P D-D 1 b$ for insensitivity to photoperiod causing early flowering and vrn-D3a for sensitivity to photoperiod causing late flowering is due to an indel polymorphism in the promoter of $P P D-D 1[14,28]$. Orthologous genes in hexaploid wheat for $V R N-A 2, V R N-B 2$, and VRN-D2 have been isolated in common wheat, but no association has been observed between a deletion in $V R N-B 2$ and flowering time in common wheat $[29,30]$.

The aim of this research was to test if the super-short life of Apogee wheat is associated with genes/alleles known to regulate flowering time in common wheat. The association of the gene alleles with segregation of flowering time was tested in a large $\mathrm{F}_{2}$ population generated by using a winter wheat cultivar 'Overland' to cross with Apogee, and the observed effects of the genes/alleles were validated in $\mathrm{F}_{3}$ populations.

\section{Results}

\section{Segregation of flowering time in $\mathrm{F}_{2}$ population}

When tested under long-day conditions and without vernalization across the experiment, Apogee flowered 29 days after planting, whereas Overland did not flower for 3 months without vernalization. When treated with low temperature at $4{ }^{\circ} \mathrm{C}$ and under long-day conditions for six weeks, Overland had earlier flowering 34 days, confirming that Overland is a winter wheat cultivar. $F_{1}$ plants that were generated from a cross between Apogee 
and Overland flowered 45 days after planting, indicating that those genes for early flowering time in Apogee were primarily dominant to those for late flowering time in Overland. A population of $858 \mathrm{~F}_{2}$ plants generated from the selfing of the $F_{1}$ plants were tested, and these $F_{2}$ plants on average flowered 48.2 days after planting, within a range from 25 days to 264 days.

The flowering time of the Apogee $\mathrm{x}$ Overland $F_{2}$ plants was continuous, and it had no clear cut-off for flowering time to distinguish between 'spring' wheat and 'winter' wheat. Plant number distribution of the $858 \mathrm{~F}_{2}$ plants showed a bias to early flowering time (Fig. 1), indicating that several genes for early flowering time are present in the population. Molecular markers for four flowering genes were identified for the causal basis of the rapid cycling of Apogee.

\section{Allelic variation in vrn-A1 and its association with flowering time}

Apogee and Overland showed no difference in the VRN-A1 promoter region by using primers VRN1AF/VRN1R, and both carry the winter $v r n-A 1$ allele. Apogee and Overland showed no difference in intron one, Ex1/C/F//Intr1/A/R3 for the non-deletion produced no product but Intr1/C/F// Intr1/AB/R amplified products of $1068 \mathrm{bp}$, the expected size for the non-deletion region. The sequencing results indicated that both Apogee and Overland carried a winter $v r n-A 1$ allele in the promoter region and intron one.

However, Apogee and Overland were detected to have differences in coding region by using primers VRNA1F4/VRN-A1R42 for a SNP in exon 4 and VRNA1F7B/VRN-A1R7 for a SNP in exon 7 to amplify the $v r n-A 1$ gene and the restriction enzyme Sph I to digest the PCR products(Fig. 2a). The sequencing results indicated that Apogee was found to carry the same vrn-A1a allele for early flowering as observed in Jagger; whereas Overland was found to have the same $v r n-A 1 b$ allele for late flowering as observed in 2174. Real time PCR result showed that Overland had two copies of vrn-A1 but Apogee had only one copy (Fig. 2e). This result confirmed that Apogee had the same allele as Jagger carrying one copy vrn-A1a and Overland had the same allele as 2174 carrying two copies $v r n-A 1 b$. The marker for SNP in exon 7 of $v r n-A 1$ was used to genotype the 858 individual plants of Apogee $\times$ Overland $F_{2}$ population.

The plants that carried the homozygous Apogee vrnA1 $a$ allele flowered, on the average, 36.8 days after planting, whereas the plants that carried the homozygous Overland $v r n-A 1 b$ allele flowered 82.5 days after planting (Table 1). The difference between the plants carrying the homozygous Apogee vrn-A1a allele and the plants carrying the heterozygous vrn-A1a/vrn-A1b alleles was 6.5 days for flowering time ( 36.8 days vs. 43.3 days), but the difference between the plants carrying the homozygous Overland $v r n-A 1 b$ allele and the plants carrying the heterozygous vrn-A1a/vrn-A1b alleles was 39.2 days for flowering time. These results indicated that the Apogee $v r n-A 1 a$ allele for early flowering was partially dominant to the Overland $v r n-A 1 b$ allele for late flowering.

\section{Allelic variation in VRN-B1 and its association with flowering time}

Apogee and Overland showed allelic variation in intron one of $V R N-B 1$. As shown in Fig. 2b, PCRs with primers Intr1/B/F//Intr1/B/R3 amplified products with 709 bp from Apogee, but Intr1/B/F//Intr1/B/R4 amplified products of $1149 \mathrm{bp}$ from Overland, indicating that the deletion was present in Apogee but not in Overland.

The average flowering time in plants homozygous for the Apogee deletion allele was 42.5 days, which was 15.4 days earlier than plants homozygous for the Overland allele (57.9 days) (Table 1). The plants carrying the homozygous Apogee deletion allele had a significantly earlier flowering time (3.6 days, $p=7.03 \mathrm{E}-08$ ) than the plants heterozygous

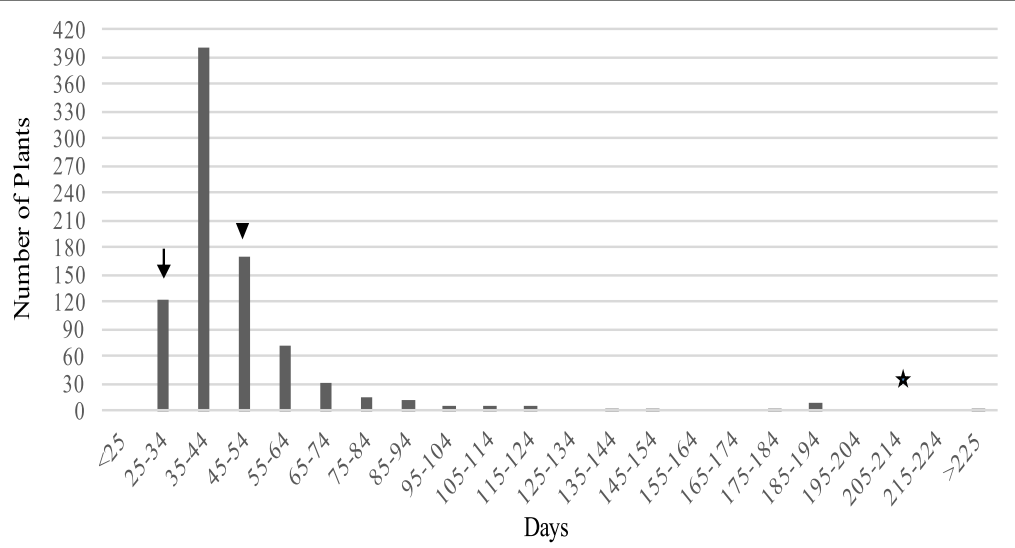

Fig. 1 Frequency distributions of plants for flowering time in $F_{2}$ population of Apogee $X$ Overland. Black arrow indicates Apogee, star indicates Overland, and black triangle indicates $F_{1}$ plant of Apogee $X$ Overland 


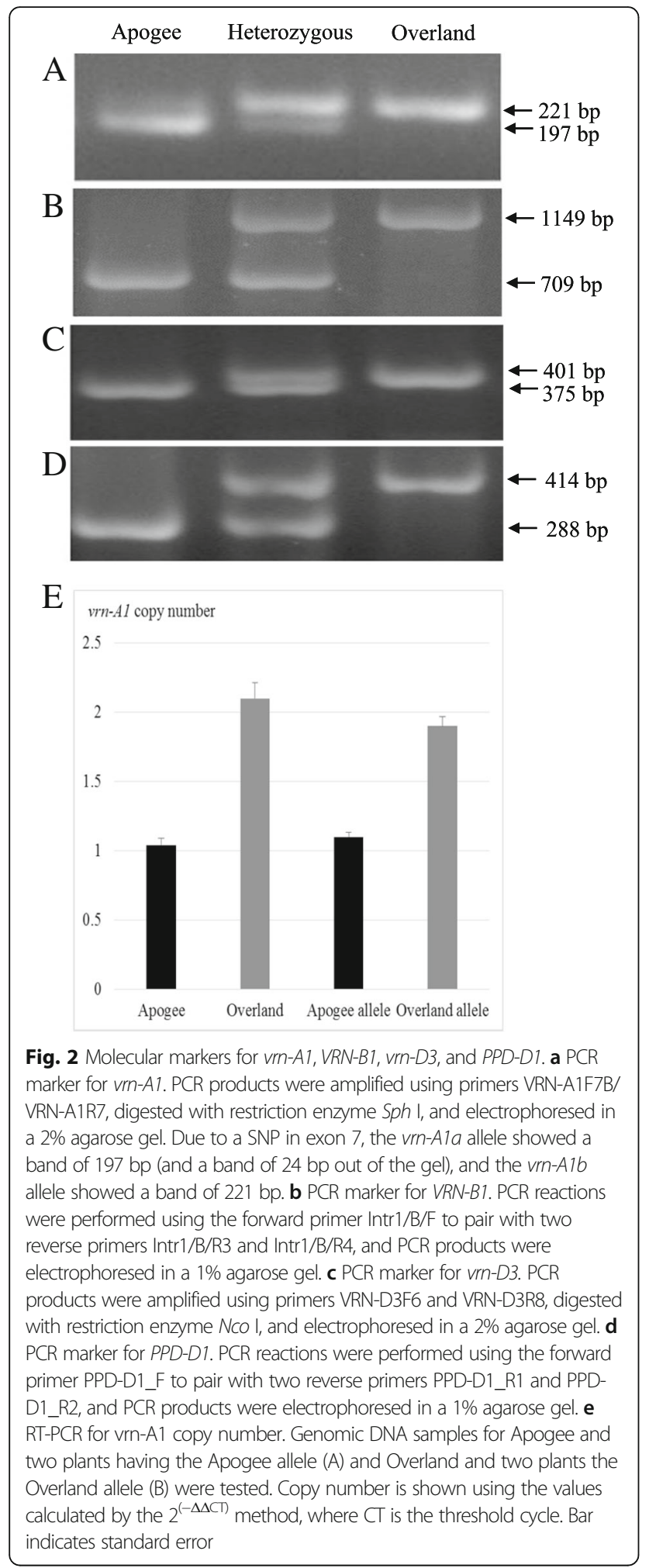

for $V R N-B 1$. The plants heterozygous for $V R N-B 1$ also were significantly earlier (11.8 days, $p=3.72 \mathrm{E}-07)$ than plants homozygous for the Overland allele. These results indicated that the Apogee deletion allele for early flowering (referred
Table 1 Comparative effects of allelic variation at vrn-A1, VRN-B1, vrn-D3, and PPD-D1 on heading date

\begin{tabular}{lllll}
\hline Gene & Allele $^{a}$ & No. plants & Heading date (days) & T-test $^{\text {b }}$ \\
\hline vrn-A1 & Apogee & 192 & 36.8 & $\mathrm{a}$ \\
& $\mathrm{H}$ & 528 & 43.3 & $\mathrm{~b}$ \\
& Overland & 138 & 82.5 & $\mathrm{c}$ \\
VRN-B1 & Apogee & 228 & 42.5 & $\mathrm{a}$ \\
& $\mathrm{H}$ & 409 & 46.1 & $\mathrm{~b}$ \\
& Overland & 221 & 57.9 & $\mathrm{C}$ \\
Vrn-D3 & Apogee & 221 & 44.5 & $\mathrm{a}$ \\
& H & 425 & 47.5 & $\mathrm{a}$ \\
& Overland & 212 & 53.4 & $\mathrm{~b}$ \\
PPD-D1 & Apogee & 247 & 45.5 & $\mathrm{a}$ \\
& H & 400 & 48 & $\mathrm{a}$ \\
& Overland & 211 & 51.8 & $\mathrm{~b}$ \\
\hline
\end{tabular}

${ }^{a}$ Apogee homozygous Apogee allele, Overland homozygous Overland allele, $H$ heterozygous alleles

bValues followed by the same letter are not significantly different at $P<0.05$, and values followed by a different letter are significantly different at $P<0.05$

to $V r n-B 1$ hereafter) was partially dominant to the Overland allele for later flowering (referred to $v r n-B 1$ hereafter).

\section{Allelic variation in vrn-D3 and its association with flowering time}

Apogee and Overland showed allelic variation in exon 3 of vrn-D3 (Fig. 2c). By using primers VRN-D3-F6/VRND3-R8 to amplify the VRN-D3 gene and following digestion with the restriction enzyme NcoI, Apogee produced two fragments, visible $375 \mathrm{bp}$ and invisible $27 \mathrm{bp}$ (out of the gel). The Apogee allele at VRN-D3 was the same as the Jagger vrn-D3a allele for early flowering time and physiological maturity; whereas Overland produced a single fragment with $401 \mathrm{bp}$ due to a deletion of $1 \mathrm{bp}$, which was the same as the $2174 v r n-D 3 b$ allele for late flowering time and physiological maturity.

Plants homozygous for the Apogee vrn-D3a allele had an average flowering time of 44.5 days, which was 8.9 days earlier than plants homozygous for the Overland vrn-D3b allele (53.4 days). The difference between the homozygous Apogee vrn-D3a allele and heterozygous vrn-D3a/vrn$D 3 b$ alleles ( 47.5 days) was not significant $(p=0.107)$, but the difference between the heterozygous vrn-D3a/vrn-D3b alleles and homozygous Overland vrn-D3b allele was significant $(p<0.001)$. These results indicated that the Apogee $v r n-D 3 a$ allele for early flowering was dominant to the Overland $v r n-D 3 b$ allele for late flowering.

\section{Allelic variation in PPD-D1 and its association with flowering time}

The diagnostic marker used to identify allelic variation in the promoter indel region of the $P P D-D 1$ gene showed that Apogee carried the photoperiod insensitive 
allele $P P D-D 1 b$ present in 2174 for early flowering but Overland carried the photoperiod sensitive allele $P P D$ D1a present in Jagger for late flowering (Fig. 2d).

Plants homozygous for the Apogee PPD-D1b allele on average flowered 45.5 days after planting, whereas plants homozygous for the Overland PPD-D1a allele on average flowered at 51.8 days, and this difference of 6.3 days between the two alleles was significant $(p<0.01)$. The difference between the homozygous Apogee PPD-D1b allele and the heterozygous PPD-D1a/PPD-D1b alleles (48 days) was not significant ( $p=0.201$ ), but the difference between heterozygous plants and homozygous plants for the Overland $P P D-D 1 a$ allele was significant $(p<0.05)$. These results indicated that $P P D-D 1 b$ for insensitivity to photoperiod (hence early flowering) was dominant to $v r n-D 3 a$ for photoperiod sensitivity (hence late flowering) as can be seen by the heterozygous alleles being not statistically different from the photoperiod insensitive homozygous plants when the population was tested under the long day conditions.

\section{Genetic effects of the four genes on flowering time} When the $858 \mathrm{~F}_{2}$ plants were genotyped, this population showed the expected 1:2:1 segregation ratio for each of the genotyped genes. Based on analysis of individual genes, the two homozygous classes of vrn-A1,VRN-B1, vrn-D3, and $P P D-D 1$ showed significant differences of 45.7, 15.4, 8.9 and 6.3 days respectively; therefore, the effect intensity of a single gene on the flowering time was ranked in the order of vrn-A1 > VRN-B1 > vrn-D3 > PPD-D1.

In addition to the accumulated effects of the four individual genes, the effects from the interactions between the four genes were analyzed in the $\mathrm{F}_{2}$ population using the factorial GLM ANOVA (Table 2). Two epistatic interactions were observed. The first epistatic interaction was between $v r n-A 1$ and $V R N-B 1$ ( $p=6.83 \mathrm{E}-107)$. The epistatic interaction was analyzed using those plants that carried homozygous allele for $v r n-A 1$ and $V R N-B 1$. In the presence of the dominant Apogee $V r n-B 1$ allele, the flowering time of 56 plants carrying the Apogee vrn-A1a allele was 35 days after planting, whereas the flowering time of 43 plants carrying the Overland $v r n-A 1 b$ allele and the dominant Apogee $V r n-B 1$ genetic background was 56.8 days after planting, showing a difference of 21.8 days (Fig. 3a). In the presence of the recessive Apogee vrn-B1 allele, the flowering time of 48 plants carrying the Apogee vrn-A1a allele was 38.6 days, whereas 27 plants carrying the Overland $v r n-A 1 b$ allele had a flowering time of 151.6 days, showing a difference of up to 113 days. Similarly, the difference between the dominant Apogee Vrn-B1 and the recessive Overland vrn-B1 was 3.6 days in the presence of the dominant Apogee vrn-A1a allele, but it was 94.8 days between the two alleles in the presence of the recessive Overland vrn-B1 allele (Fig. 3a).

The second epistatic interaction was between vrn-A1 and vrn-D3 $(p=6.33 \mathrm{E}-7)$. Due to the presence of interaction between $v r n-A 1$ and $V R N-B 1$, the interactions $v r n$ $A 1$ and $v r n-D 3$ were analyzed under different $V R N-B 1$ genetic backgrounds. Regardless of the presence or absence of $V R N-B 1$, the $v r n-D 3$ effect within the dominant Apogee vrn-A1a allele was 5.1 days (34.9 days for $v r n$ $D 3 a$ in 56 plants compared with 40 days for $v r n-D 3 b$ in 38 plants), which was significant $(p<0.01)$ (Fig. 3b). The

Table 2 Analyses on interactions among vrn-A1, VRN-B1, vrn-D3, and PPD-D1

\begin{tabular}{|c|c|c|c|c|c|c|}
\hline Source & DF & Sum of squares & Mean square & F Value & $\operatorname{Pr}>\mathrm{F}$ & R2 (\%) \\
\hline$v r n-A 1$ & 2 & $199,978.4759$ & $99,989.2379$ & 577.45 & $<.0001$ & $38 \%$ \\
\hline$V R N-B 1$ & 2 & $40,238.9184$ & $20,119.4592$ & 116.19 & $<.0001$ & $8 \%$ \\
\hline$v r n-A 1 * V R N-B 1$ & 4 & $128,602.4832$ & $32,150.6208$ & 185.67 & $<.0001$ & $24 \%$ \\
\hline PPD-D1 & 2 & 4085.9387 & 2042.9694 & 11.8 & $<.0001$ & $1 \%$ \\
\hline$v r n-A 1 * P P D-D 1$ & 4 & 2213.353 & 553.3382 & 3.2 & 0.0129 & $0 \%$ \\
\hline$V R N-B 1 * P P D-D 1$ & 4 & 314.2033 & 78.5508 & 0.45 & 0.7698 & $0 \%$ \\
\hline$v r n-A 1 * V R N-B 1 * P P D-D 1$ & 8 & 3239.9174 & 404.9897 & 2.34 & 0.0174 & $1 \%$ \\
\hline$v r n-D 3$ & 2 & 4159.1734 & 2079.5867 & 12.01 & $<.0001$ & $1 \%$ \\
\hline$v r n-A 1 * v r n-D 3$ & 4 & 4007.5511 & 1001.8878 & 5.79 & 0.0001 & $1 \%$ \\
\hline$V R N-B 1^{*}$ vrn-D3 & 4 & 1430.9048 & 357.7262 & 2.07 & 0.0835 & $0 \%$ \\
\hline$v r n-A 1^{*} V R N-B 1^{*} v r n-D 3$ & 8 & 3339.6495 & 417.4562 & 2.41 & 0.0142 & $1 \%$ \\
\hline$P P D-D 1 * v r n-D 3$ & 4 & 415.8344 & 103.9586 & 0.6 & 0.6625 & $0 \%$ \\
\hline$v r n-A 1^{*} P P D-D 1 * v r n-D 3$ & 8 & 1560.2604 & 195.0326 & 1.13 & 0.3429 & $0 \%$ \\
\hline$V R N-B 1^{*} P P D-D 1^{*} v r n-D 3$ & 8 & 944.0415 & 118.0052 & 0.68 & 0.7082 & $0 \%$ \\
\hline$v r n-A 1 * V R N-B 1 * P P D-D 1 * v r n-D 3$ & 14 & 2447.9668 & 174.8548 & 1.01 & 0.441 & $0 \%$ \\
\hline Error & 779 & $134,889.9808$ & 173.1579 & & & \\
\hline Corrected Total & 857 & $531,868.6527$ & & & & \\
\hline
\end{tabular}


A

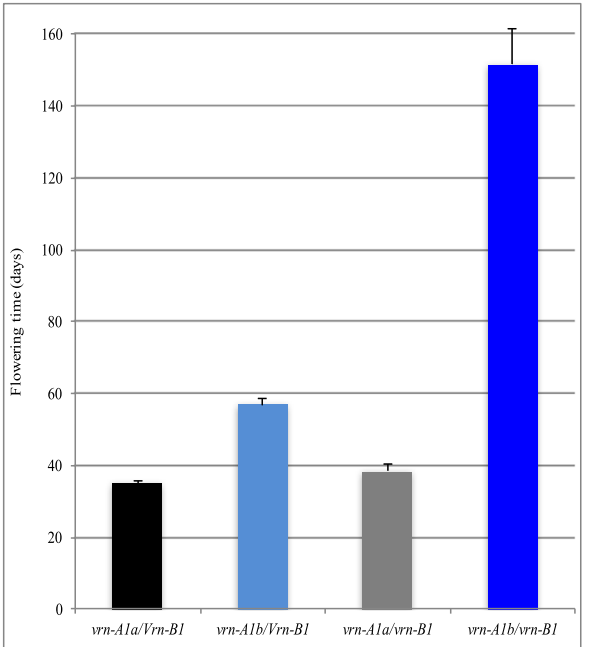

$\mathrm{C}$

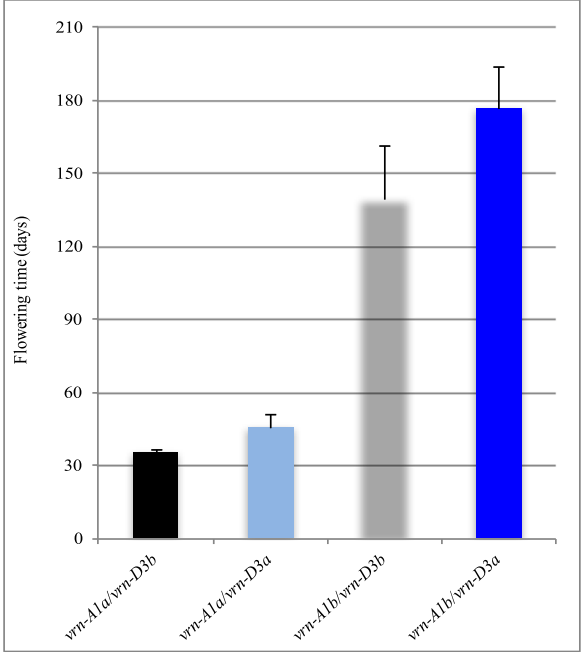

B

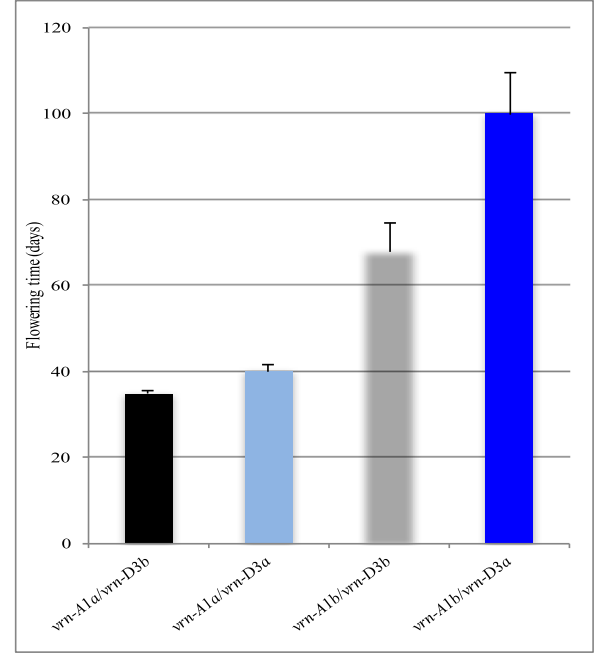

$\mathrm{D}$

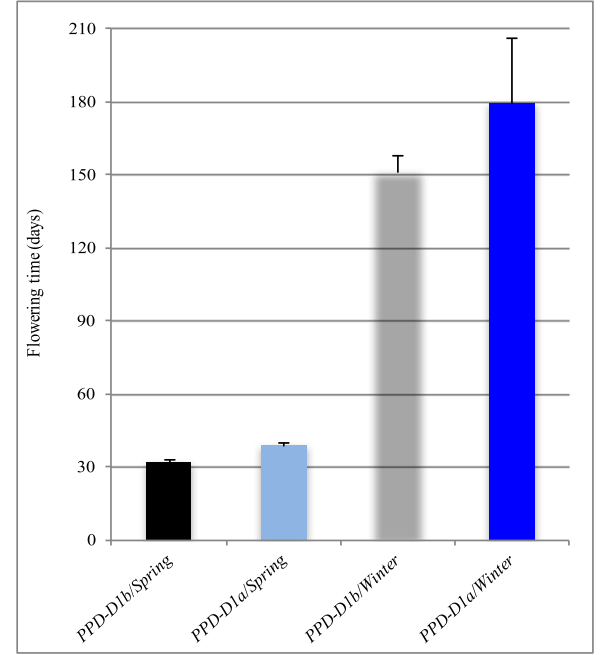

Fig. 3 Interactive effects of flowering genes. a Interactions between vrn-A1 and VRN-B1. Plants are classified into four genotypic classes and compared for flowering time, dominant vrn-Ala and dominant $V r n-B 1$, dominant $v r n-A 1 a$ but recessive $v r n-B 1$, recessive $v r n-A 1 b$ but dominant $V r n-B 1$, and recessive $v r n-A 1 b$ and recessive $v r n-B 1$. $\mathbf{b}$ Interactions between $v r n-A 1$ and $v r n-D 3$. All plants are classified into four genotypic classes regardless of allele status of VRN-B1 or PPD-D1. $\mathbf{c}$ Interactions between $v r n-A 1$ and $v r n-D 3$ in the presence of recessive vrn-B1 allele. All plants that have dominant $V r n-B 1$ allele are classified into four genotypic classes and compared for flowering time, dominant vrn-A1a and dominant vrn-D3a, dominant $v r n-A l a$ but recessive $v r n-D 3 b$, recessive $v r n-A 1 b$ but dominant $v r n-D 3 a$, and recessive $v r n-A 1 b$ and recessive $v r n-D 3 b$. $\mathbf{d}$ Effects of PPD$D 1$ under different genetic backgrounds. Spring indicates the dominant allele for vrn-Ala and Vrn-B1, and winter indicates the recessive allele for vrn-A1b and vrn-B1

vrn-D3 effect within the recessive Overland $v r n-A 1$ genetic background was 31.8 days (68 days for vrn-D3a in 38 plants vs. 99.8 days for $v r n-D 3 b$ in 34 plants), which was also significant $(p<0.01)$ (Fig. 3b). However, vrn-A1 and $v r n-D 3$ interaction was not always detectable under different VRN-B1 backgrounds. When the dominant Vrn-B1 was present, the vrn-D3 effect within the dominant Apogee $v r n-A 1 a$ allele was 2.6 days ( 34.2 days for $v r n-D 3 a$ vs. 36.8 days for $v r n-D 3 b)$, which was not significant $(p=0.142)$. However, the vrn-D3 effect within the recessive Overland vrn-A1 genetic background was 14.1 days
(51.1 days for vrn-D3a vs. 65.2 days for vrn-D3b), which was significant $(p<0.05)$. Conversely, when the dominant $V r n-B 1$ was absent, the vrn-D3 effect within the dominant Apogee $v r n-A 1 a$ allele was 10.6 days (35 days for $v r n-D 3 a$ vs. 45.6 days for $v r n-D 3 b)$, which was significant $(p<0.05)$ (Fig. 3c). However, the vrn-D3 effect within the recessive Overland vrn-A1 genetic background was 37.3 days (139.4 days for vrn-D3a vs. 176.7 days for vrn$D 3 b)$, which was not significant $(p=0.206)$ due to a large variation in flowering time in winter plants without vernalization (Fig. 3c). 
Significant interactions were also detectable among three genes, vrn-A1, VRN-B1, and vrn-D3, and among three genes, vrn-A1, VRN-B1, and PPD-D1 ( $p=0.0174)$. When both vrn-A1a and Vrn-B1 were fixed at a dominant allele for spring type, the plants homozygous for the $P P D-D 1 b$ allele flowered 31.8 days after planting and the plants homozygous for the $P P D-D 1 a$ allele flowered 38.4 days after planting. The difference between the two alleles was 6.6 days and significant ( $p=8.14 \mathrm{E}-05)$ (Fig. $3 d)$. However, when both $v r n-A 1 b$ and $v r n-B 1$ were fixed at a recessive allele for winter type, the plants homozygous for the $P P D-D 1 b$ allele flowered 151 days after planting and $P P D-D 1 b$ the plants carrying homozygous $P P D-D 1 a$ allele flowered 179.2 days after planting. The difference between the two alleles was 28.2 days, but it was not significant $(p=0.392)$ again due to large variation among the plants behaving as winter wheat (Fig. $3 \mathrm{~d})$. Therefore, the effects of PPD-D1 was dependent on the genetic background.

\section{Validation of the genetic effects of the genes/alleles on flowering time in $\mathrm{F}_{\mathbf{3}}$ populations}

A subset of $203 \mathrm{~F}_{2: 3}$ lines were used to validate the genetic effects of the four flowering time. These $F_{3}$ lines, each derived from the same $\mathrm{F}_{2}$ line, were tested in two different locations. The genetic effects of the vrn-A1 gene on flowering time were observed in these $F_{3}$ lines at both Oklahoma State University (OSU) and University of Nebraska in Lincoln (UNL) (Fig. 4a). However, the genetic effects of the $V R N-B 1$ gene and the $v r n-D 3$ gene were detectable at OSU but not at UNL, whereas the genetic effects of the PPD-D1 gene were detectable at UNL but not at OSU (Fig. 4a).

The total effects of the four individual genes together with their interactive effects, explained approximately $75 \%$ of the total phenotypic variation in the $F_{2}$ population (Table 2). To test if any other major genes affected the segregation of flowering time, $203 \mathrm{~F}_{2: 3}$ lines were genotyped using genotyping-by-sequencing (GBS). A total of 61,217 SNP markers was identified in the population. Further, filtering with appropriate filtering criteria (see Methods) and polymorphism for both the parents, 1969 high-quality SNPs were used to develop linkage maps. These high-quality markers were then assembled into 22 linkage groups that were assigned to 21 chromosomes and an uncharacterized group. A major QTL peak at the $v r n-A 1$ gene was observed for flowering time in the $\mathrm{F}_{2}$ population and $F_{3}$ populations (Fig. 4b). No other QTL was observed in the GBS-genotyped population.

\section{Discussion}

The cumulative effects of the early alleles for the four flowering time genes result in the super short life cycle of Apogee.
Apogee wheat was found to have an early allele for all of four flowering genes identified in this study, as Apogee was crossed with winter cultivar Overland and the resulting $\mathrm{F}_{2}$ population was tested under constant warm temperature and long day conditions. The allele combination of the four genes resulted in a genotype that flowered as early as 25 days after planting. All four genes are related to the genes known for responses to vernalization and photoperiod, the two external cues that are regarded as the most important factors regulating wheat development.

However, it was surprising that that Apogee had the same $v r n-A 1 a$ allele as observed in winter wheat cultivar Jagger. The flowering genes $V R N-A 1$ were cloned based on phenotypic differences between spring wheat carrying the dominant $V r n-A 1$ and winter wheat carrying the recessive $v r n-A 1$ allele [10]. Moreover, $v r n-A 1$ was found to control variation in vernalization requirement duration between two winter wheat cultivars, Jagger carrying the $v r n-A 1 a$ allele for less vernalization requirement and 2174 carrying the $v r n-A 1 b$ for more vernalization requirement [23]. Surprisingly, Apogee was found to have the $v r n-A 1 a$ allele for early flowering, which is the same allele as observed in Jagger, whereas Overland was found to have the $v r n-A 1 b$ allele for late flowering, which is the same allele as observed in 2174. This observation also supported our report that the early flowering conferred by $v r n-A 1$ was due to the protein form of $v r n$ $A 1 a$ but not due to two copies of vrn-A1b [23]. It would be intriguing to know how the vernalization requirement by the $v r n-A 1 a$ allele in Apogee is overcome.

It was also surprising that Apogee had the same vrn-D3a allele as observed in winter wheat cultivar Jagger. The wheat $V R N-B 3$ gene was cloned using a population generated from two cultivars, CS and Hope, both of which have a dominant Vrn-D1 allele, and the difference between the $V r n-B 3$ and $v r n-B 3$ relies on an indel polymorphism in the promoter region involved in the regulation of the $V R N-B 3$ transcript levels [11]. Furthermore, vrn-D3 was identified to be associated with allelic variation for flowering time among winter wheat cultivars [26-28]. The difference between the vrn-D3a in Apogee like Jagger and $v r n-D 3 b$ in Overland like 2174 relies on a SNP that results in a frame shift involving 81 amino acids [26].

\section{Comparative effects of different flowering genes in the same population}

This is the first study that four genes were analyzed for their effects on flowering time in the same population in hexaploid wheat. In the Apogee $\mathrm{x}$ Overland $\mathrm{F}_{2}$ population, a single $v r n-A 1 a$ allele was able to flower as early as 30 days after planting. Due to the strongest effect of the $\mathrm{Vrn}-\mathrm{Ala}$ allele, it has been incorporated into spring wheat cultivars in Canadian breeding programs to provide frost avoidance in short-season environments [31]. Conversely, a deletion 

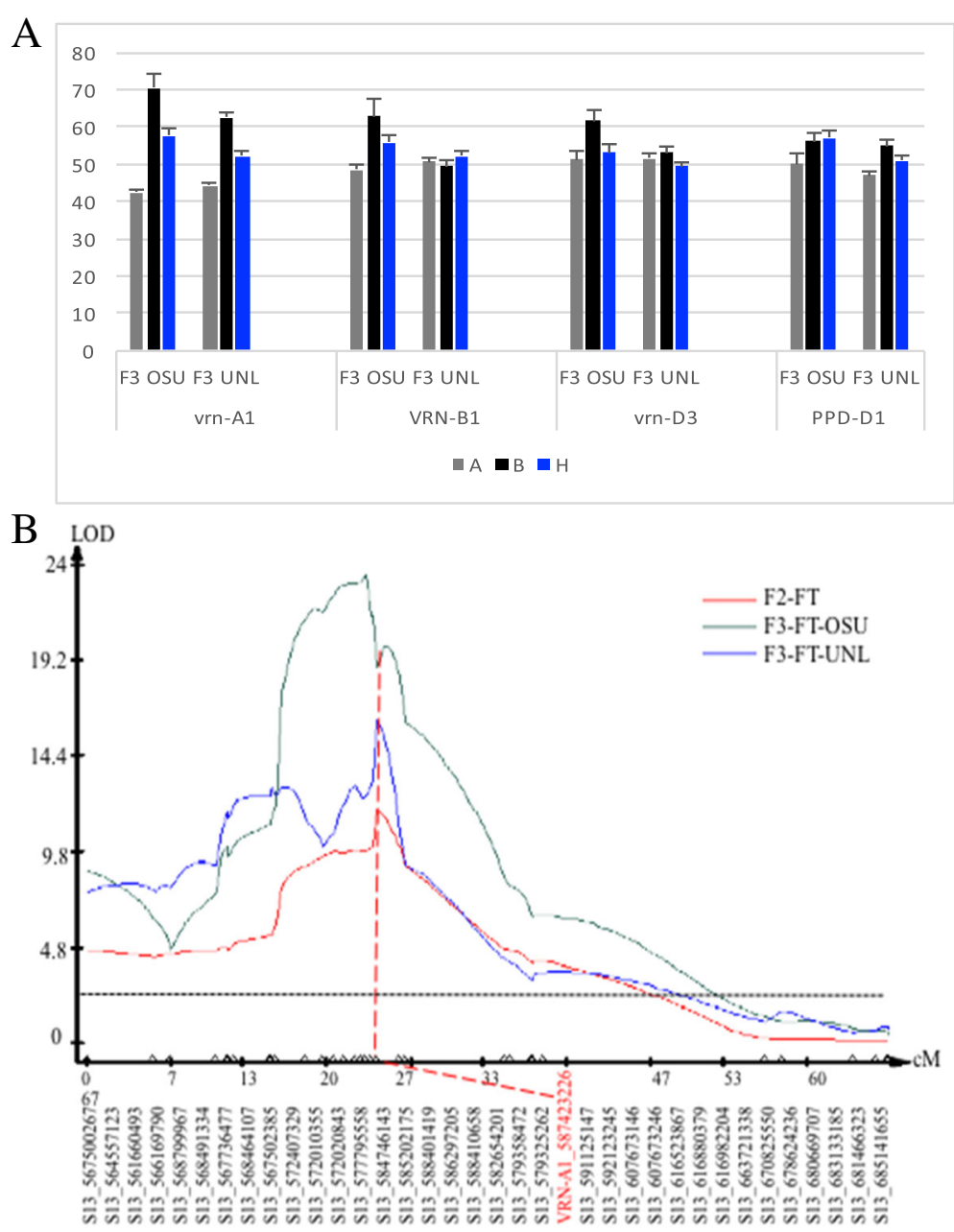

Fig. 4 Mapping of flowering genes in Apogee $x$ Overland populations. a Genetic effects of individual genes on flowering time in $F_{3}$ populations. A indicates the Apogee allele, B indicates the Overland allele, and $\mathrm{H}$ indicates the heterozygous allele. $\mathbf{b}$ The major QTL at the vrn-A1 locus. The number of a GBS marker indicates the physical location of the marker in the wheat genome sequence of Chinese Spring released in the International Wheat Genome Sequencing Consortium (IWGSC). The vertical dotted line indicates the logarithm of the odds (LOD) significance threshold of 2.5. The vrn-A1 gene assembled in this group is highlighted in red

of TmVRN1 in T. monococcum results in a never-flowering wheat plant (mvp) [32]. These studies have demonstrated the powerful effect of $V R N-A 1$ on flowering time in both spring wheat and winter wheat backgrounds.

The comparative effects of different genes on flowering time in wheat were reported in previous studies. The effect intensity of the three $V R N-1$ genes was ranked as $V r n-A 1>V r n-B 1>V r n-D 1$, based on analyses on Triple Dirk isogenic lines [33] and in Chinese wheat cultivars [34]. This study presented experimental evidence for the greater effect of $v r n-A 1 a$ than $V r n-B 1$ on flowering time in a single segregating population.

\section{Allelic variation in vrn-A1a and its epistatic interaction with Vrn-B1}

In a previous study, the spring and dominant allele $\mathrm{Vrn}$ A1a was reported to cause earlier transcription of the recessive $v r n-B 1$ allele in the Triple Dirk line TDD, forming of a positive feedback loop that coordinates the transcription of the different $V R N-1$ alleles during flowering initiation [35]. In this study, the winter but dominant vrn-A1a allele was also epistatic to $V R N-B 1$. The effect of $V R N-B 1$ on flowering time was 3.6 days in the presence of the Apogee vrn-A1a allele (vrn-A1a/Vrn-B1 vs. vrn-A1a/vrn-B1) but this effect was increased to 94.8 days in the presence of the Overland $v r n-A 1 b$ allele $(v r n-A 1 b / V r n-B 1$ vs. vrn- $A 1 b$ / $v r n-B 1)$. The effect of the dominant $V r n-B 1$ allele on $v r n-$ $A 1$ was also observed in the phenotype. The effect of $v r n$ $A 1$ on flowering time was 21.8 days in the presence of the Apogee $V r n-B 1$ allele (vrn-A1a/Vrn-B1 vs. vrn-A1b/Vrn$B 1)$ but this effect was increased to 113 days in the presence of the Overland vrn-B1 allele (vrn-A1a/vrn-B1 vs. vrn- $A 1 b$ / $v r n-B 1 b)$. Since the plants carrying the dominant $v r n-A 1 a$ allele but the recessive $v r n-B 1$ allele had earlier flowering 
time (35 days) than the plants carrying the dominant $\mathrm{Vrn}$ $B 1$ allele but the recessive $v r n-A 1 b$ allele, it is more likely that vrn1-A1a was epistatic to $V R N-B 1$ in the Apogee and Overland $\mathrm{F}_{2}$ population. This study indicated that the accelerated effect of the vrn-Ala allele on flowering time was detectable under different genetic backgrounds in wheat.

\section{Accelerated effect of the dominant PPD-D1b and vrn-3a allele for flowering but not for the developmental transition}

Vernalization and photoperiod genes are believed to interactively regulate flowering time in temperate cereal crops [27, 36-40], but no direct evidence is available for interactions of multiple flowering time genes in hexaploid wheat. Results in this study indicated that $P P D-D 1 b$, which is insensitive to photoperiod, can be used to accelerate flowering in spring plants that have been induced to flower by $v r n-A 1 a$ or $V r n-B 1$. However, no significant effect was observed when $P P D-D 1 b$ was placed in the genetic backgrounds of recessive $v r n-A 1 b$ and $v r n-B 1$. When $v r n-A 1 a$ and $V r n-B 1$ were fixed in a plant for an early flowering allele, the promotion effect of the vrn-D3a allele was not detectable, because this plant flowered extremely early already. When $v r n-A 1 b$ and $v r n-B 1$ were fixed in a plant for a late flowering allele, the effect of the $v r n-D 3 a$ allele was not significant either, because vrn-D3a alone was insufficient to initiate the transition from the vegetative to reproductive development in winter wheat without vernalization. The vrn-D3 haplotype was functionally characterized in the Jagger $\times 2174$ RIL population, in which that $v r n-D 3 a$ was found to have accelerated flowering and physiological maturity dates but no significant effect on initial stem elongation, a trait best representing the developmental transition from vegetative to reproductive development [40]. This conclusion was also true in another winter wheat population generated from two winter cultivars Intrada and Cimarron [27]. The same vrn-D3 haplotype was also reported to have significant effect on flowering time in a large collection of diverse germplasm [26].

Based on these observations, a model for regulating node sites of the four flowering genes in the vernalization pathway is established (Fig. 5). In this model, vrn-A1a replaces vernalization to directly induce the developmental transition. Vrn-B1 is able to induce this transition, but it acts later than vrn-A1a and is also accelerated by vrn-A1a due to the presence of a positive feedback loop. Under the experimental conditions tested in this study, $P P D-D 1 b$ and $v r n-D 3 a$ are able to accelerate flowering only after the developmental transition has been initiated by $v r n-A 1 a$ or $V r n-B 1$ in spring plants. In this model, vrn-D3a acts in the downstream of $V r n-A 1$, which seems contradictory to a previous model, in which $V r n-A 1$ acts in the downstream of $V r n-B 3$ in the vernalization pathway [11]. However, $v r n-D 3 a$ and $V r n-B 3$ may have different mechanisms in regulation of flowering time under different genetic backgrounds in wheat.

\section{Conclusion}

Apogee wheat is able to flower one month after planting and without vernalization requirement, so each generation will be 2 months or less and we can grow up to 6 generations per year which will greatly shorten the breeding cycle. Conversely, a winter wheat cultivar takes at least 4 to 5 months per generation, so only two (or at most three) generations can be grown per year. When winter wheat cultivars are crossed and backcrossed with Apogee, the resulting backcrossed lines will be selected for reduced generation time using molecular tools for the Apogee alleles for the four flowering genes. Such rapid cycling lines will not require vernalization to induce flowering and will be selected for traits of interest such as disease or pest resistance, high yield, or end-use quality. The elite breeding lines will then be crossed to the winter wheat recurrent parent used to create the rapid cycling lines to recover the winter growth habit, thus creating adapted backcross derived lines quickly and efficiently, and accelerating winter wheat breeding schemes.

\section{Methods}

Apogee (PI 592742) is a full-dwarf hard red spring wheat cultivar (pedigree: Parula/Super dwarf) and released by the Utah Agric. Exp. Station in cooperation with NASA in 1996. 'Overland' (PI 647959) is hard red winter wheat [pedigree: Millennium sib//(ND8974) Seward/Archer)] that was developed by the Nebraska Agriculture Experiment Station and released in 2007. Apogee and Overland were tested three times in a greenhouse conditioned with long days $\left(16 \mathrm{~h}\right.$ for light at $25 \pm 2{ }^{\circ} \mathrm{C}$, and $8 \mathrm{~h}$ for darkness at $20 \pm 2{ }^{\circ} \mathrm{C}$ ) during the whole life cycle, located on the Stillwater campus of Oklahoma State University (OSU). In addition to natural sunlight, high-pressure sodium lamps were used to provide supplemental lighting for nights and cloudy days. Apogee flowered about 30 days after planting, whereas Overland did not flower within 6 months unless it was vernalized. Overland was vernalized at $4{ }^{\circ} \mathrm{C}$ and under long days. Apogee was crossed with Overland to create 20 hybrid seeds. The $F_{1}$ seeds were self-pollinated and used to generate a population of $858 \mathrm{~F}_{2}$ plants.

All of the $F_{2}$ plants were not vernalized to identify genes associated with early flowering genes in Apogee without requirement of vernalization. An $\mathrm{F}_{2}$ population consisting of up to 858 plantswas tested in this study, and the large population size should have replication of each genotypic class, necessary for statistical comparisons. A total of $203 \mathrm{~F}_{2: 3}$ lines were planted in February 2015 and tested at OSU and University of Nebraska in Lincoln (UNL) to validate the effects of flowering time genes. The flowering time of a plant was recorded when 


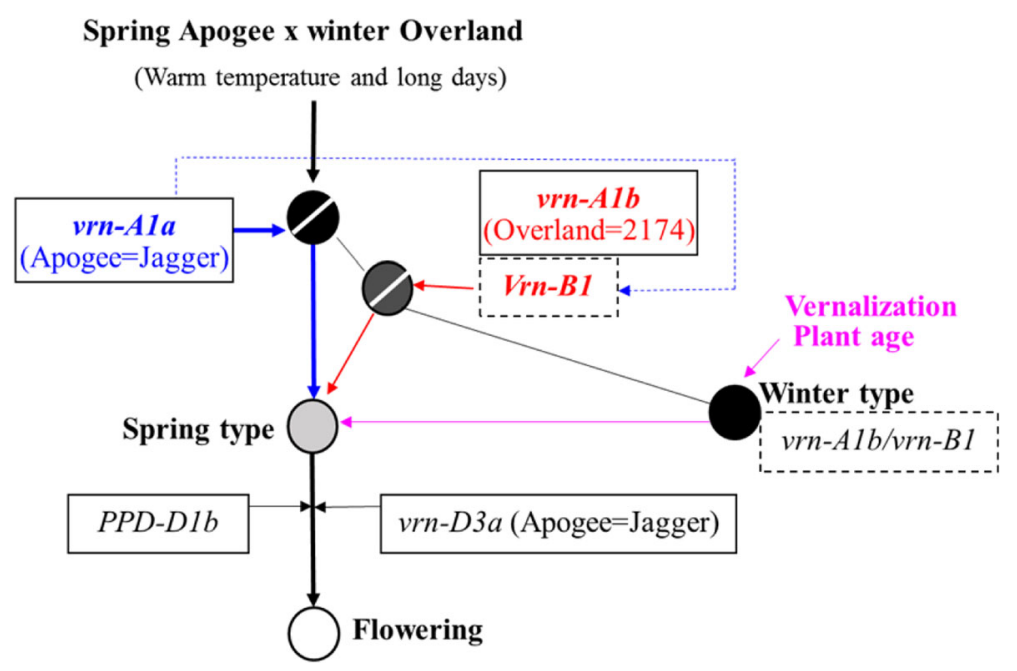

Fig. 5 An updated genetic model for the wheat flowering pathway. Thin arrows indicate promotion. When grown under warm temperature and long day condition, the Apogee $\times$ Overland $F_{2}$ population are segregated to into different genotypes; the plants that carry dominant vrn-A1a allele will behave as spring type and flower first (flowering signals transited in the pathway indicted in blue), the plants that carry dominant Vrn-B1 allele will behave as spring type and flower later (flowering signals transited in the pathway indicated in red), because Vrn-B1 has less power than $v r n-A 1 a$, and $V r n-B 1$ is promoted by $v r n-A 1 a$ in a positive feedback loop; the plants that carry recessive alleles for both $v r n-A 1 b$ and $v r n-B 1$ will behave as winter type and will not flower unless vernalization is provided or flower with plant age. PPD-D1b and vrn-D3a will accelerate flowering in the spring plants that have been induced to flower by vrn-A1a/Vrn-B1 or the winter plants that have been vernalized

the first spike of the plant was emerging from the flag leaf sheath of the main stem or a primary tiller. Flowering time was scored for each plant of the population.

Genomic DNA was extracted from parental lines and the $858 \mathrm{~F}_{2}$ individual plants using the method as previously described [41]. PCR markers for genes known to regulate flowering time were used to identify polymorphism between Apogee and Overland, and primer sets used for the gene markers are listed in Additional file 1: Table S1. The protocols for PCR markers for each gene were cited from previous studies. Those markers that showed allelic variation between Apogee and Overland were used to genotype $858 \mathrm{~F}_{2}$ plants, and those markers that showed no difference were not further analyzed. Details for the four genes that showed allelic variation between Apogee and Overland are elaborated below for convenience.

\section{VRN-A1}

Five pairs of primers were used to test polymorphisms in VRN-A1 between Apogee and Overland. The first primer set, VRN1AF/VRN1R, was used to detect indel polymorphisms in the VRN-A1 promoter region. The second and third primer sets were used to detect the indel polymorphism in intron 1 in $V R N-A 1, \mathrm{Ex} 1 / \mathrm{C} / \mathrm{F} / /$ Intr1/A/R3 for the deletion in the Vrn-A1c allele and Intr1/C/F//Intr1/AB/R for the non-deletion in the vrn$A 1$ allele [24]. The fourth and fifth primer sets were used to detect the difference in coding region, VRN-A1F4/ VRN-A1R42 for a SNP in exon 4 [40] and VRN-A1F7B/ VRN-A1R7 for a SNP in exon 7 [23].

\section{VRN-B1}

Two pairs of primers were used to detect the indel polymorphism in intron one in $V R N-B 1$, Intr1/B/F// Intr1/B/R3 for the deletion in the $V r n-B 1$ allele and Intr1/B/F//Intr1/B/R4 for the non-deletion in the $v r n$ B1 allele [24]. Primers Intr1/B/F//Intr1/B/R3 amplified products with a band of $709 \mathrm{bp}$, and Intr1/B/F//Intr1/ B/R4 amplified products with a band of $1149 \mathrm{bp}$. The forward primer and the two reverse primers for the same progeny sample were used to amplify the homozygous or heterozygous $V R N-B 1$ alleles in two different reactions, but the PCR products were electrophoresed in the same gel well.

\section{VRN-D3}

The primer set VRN-D3-F6/VRN-D3-R8 was used to test allelic variation in $V R N-D 3$. Products were digested with restriction enzyme NcoI, producing polymorphic DNA fragments due to a $1 \mathrm{bp}$ indel or $(\mathrm{G})_{3 \text { or4 }}$ difference in VRN-D3 coding region, $375 \mathrm{bp}$ and $27 \mathrm{bp}$ fragments for vrn-D3a allele and $401 \mathrm{bp}$ for the vrn-D3b allele [28].

\section{PPD-D1}

One forward primer PPD-D1_F was paired with two reverse primers PPD-D1_R1 and PPD-D1_R2 to simultaneously amplify two PPD alleles in a one shot PCR reaction [14]. One allele is for deletion, producing a $288 \mathrm{bp}$ fragment from the photoperiod insensitive allele, and the other is for non-deletion, producing a $414 \mathrm{bp}$ fragment from the photoperiod sensitive allele. 
Real time-PCR was used to determine VRN-A1 copy number by the SYBR Green PCR Master Mix, and TaCO2 was used as an endogenous control. Primers for vrn-A1 and for $\mathrm{TaCO} 2$ control were cited from previous studies [35].

The gene interactions were assessed using the Factorial analysis of variance (ANOVA) of the General Linear Model (GLM) in SAS 9.4 (SAS Institute, Raleigh, NC). The epistatic interactions between two loci were analyzed using the program EPISTACY in SAS 9.4 [42].

The $203 \mathrm{~F}_{3}$ lines were genotyped using GBS at the Wheat Genetics and Germplasm Improvement Laboratory at Kansas State University (http://www.wheatgenetics.org). The SNP calls were made using the TASSEL v5.2.30 software (Glaubitz et al. 2014) with default setting except for two parameters to increase the SNP calling stringency. First, minimum quality score within the barcode to be accepted was changed from default value of 0 to 20 . Second, minimum count of reads for a tag to be output for alignment against the reference genome was changed from default value of 1 to 5 . The reference genome v0.4 of the bread wheat variety 'Chinese spring' was utilized for making SNP calls. The SNP markers were filtered to exclude markers with missing percentage greater than $20 \%$ and minor allele frequency less than 0.2. The SNPs were then converted from a nucleotidebased format to parent-based format using GenosToABHPlugin in TASSEL for linkage map development. Based on the conserved locations of the GBS SNP markers, these linkage groups were assigned to 19 of the 21 chromosomes in hexaploid wheat. On the basis of whole-genome QTL scanning using Interval Mapping (IM) analysis, the QTL for flowering time was screened.

Terms for genes/alleles are referred to, VRN-A1 for a common allele for either dominant or recessive allele, $V r n-A 1$ for the dominant allele in spring wheat, vrn-A1a for a recessive allele present in winter wheat Jagger for early flowering and $v r n-A 1 b$ for a recessive allele present in winter wheat 2174 for late flowering; $V r n-3$ for the dominant allele in spring wheat, $v r n-D 3 a$ for a recessive allele present in Jagger for early flowering and $v r n-A 1 b$ for a recessive allele present in 2174 for late flowering; $\mathrm{Vrn}$ $B 1$ for early flowering in spring wheat, and $v r n-B 1$ for late flowering in winter wheat; $P P D-D 1 a$ for photoperiod sensitive and late flowering in Jagger and $P P D-D 1 b$ for photoperiod insensitive and early flowering in 2174 .

\section{Additional file}

Additional file 1: Table S1. Primers used in PCR reactions for identification of allelic variation at vrn-A1, VRN-B1, vrn-D3, and PPD-D1. (DOCX 18 kb)

\section{Abbreviations}

EPS: Earliness per se (EPS); GBS: Genotyping-by-sequencing; MITE: Miniature inverted-repeat transposable element; PCR: Polymerase chain reaction; PPD: Photoperiod response; SNP: Single nucleotide polymorphism; VRN: Vernalization

\section{Acknowledgements}

The authors thank Dr. Jesse Poland for genotyping the population using a GBS approach.

\section{Author contributions}

$\mathrm{GL}, \mathrm{RB}, \mathrm{CP}, \mathrm{TH}$, and FM conducted marker development, genotyping, and mapping; $V B$ processed the genotyping-by-sequencing data and performed SNP calling; PSB and LY conceived the project and designed the experiments. All authors analyzed data and reviewed the manuscript. GL, RB, CP, PSB and LY wrote the manuscript. All authors read and approved the final manuscript.

\section{Funding}

This study was partially funded by the Oklahoma Agricultural Experiment Station and the Nebraska Agricultural Experiment Station.

\section{Availability of data and materials}

The seeds used and/or analyzed during the current study available from the corresponding author on reasonable request.

Ethics approval and consent to participate

Not applicable.

\section{Consent for publication}

Not applicable.

\section{Competing interests}

The authors declare that they have no competing interests.

\section{Publisher's Note}

Springer Nature remains neutral with regard to jurisdictional claims in published maps and institutional affiliations.

\section{Author details}

'Department of Plant and Soil Sciences, Oklahoma State University, Stillwater OK74078, USA. ${ }^{2}$ Department of Agronomy and Horticulture, University of Nebraska-Lincoln, Lincoln, NE 68583, USA. ${ }^{3}$ Wheat, Peanut and Other Field Crops Research, USDA-ARS, 1301 N Western RD, Stillwater, OK 74075-2714, USA. Institute of Grain Crops, Xinjiang Academy of Agricultural Sciences, Urumqi 830091, People's Republic of China. ${ }^{5}$ College of Life Science, Northwest A \& F University, Yangling, Shaanxi 712100, People's Republic of China.

Received: 4 May 2017 Accepted: 25 October 2017

Published online: 31 October 2017

References

1. Fu B, Nelson PE. Conditions and constraints of food processing in space. Food Technol. 1994:48:113-27.

2. Zasypkin DV, Lee TC. Food processing on a space station: feasibility and opportunities. Life Support Biosphere Sci. 1999;6:39-52.

3. Bugbee BG, Koerner G, Albrechtsen R, Dewey W, Clawson S. Registration of cultivars. Crop Sci. 1997;37:626-1141.

4. Bugbee BG, Salisbury FB. Exploring the limits of crop productivity. Plant Physiol. 1988;88:869-78.

5. Kato K, Yamagata H. Method for evaluation of chilling requirement and narrow-sense earliness of wheat cultivars. Jpn J Breed. 1988;38:172-86.

6. Laurie DA, Pratchett N, Bezant JH, Snape JW. RFLP mapping of five major genes and eight quantitative trait loci controlling flowering time in a winter x spring barley (Hordeum vulgare L.) cross. Genome. 1995;38:575-85.

7. Snape JW, Butterworth K, Whitechurch E, Worland AJ. Waiting for fine times: genetics of flowering time in wheat. Euphytica. 2001;119:185-90.

8. Flood RG, Halloran GM. Genetics and physiology of vernalization response in wheat. Adv Agron. 1986;39:87-125.

9. Griffiths S, Dunford RP, Coupland G, Laurie DA. The evolution of CONSTANS-like gene families in barley, rice, and Arabidopsis. Plant Physiol. 2003;131:1855-67.

10. Yan L, Loukoianov A, Tranquilli G, Helguera M, Fahima T, Dubcovsky J. Positional cloning of the wheat vernalization gene VRN1. Proc Natl Acad Sci U S A. 2003; 100:6263-8.

11. Yan L, Fu D, Li C, Blechl A, Tranquilli G, Bonafede M, Sanchez A, Valarik M, Yasuda S, Dubcovsky J. From the cover: the wheat and barley vernalization gene VRN3 is an orthologue of FT. Prod Natl Acad Sci USA. 2006;103:19581-6. 
12. Kippes N, Debernardi JM, Vasquez-Gross HA, Akpinar BA, Budak H, Kato K, Chao S, Akhunov E, Dubcovsky J. Identification of the VERNALIZATION 4 gene reveals the origin of spring growth habit in ancient wheats from South Asia. Proc Natl Acad Sci U S A. 2015;112:5401-10.

13. Yan L, Loukoianov A, Tranquilli G, Blechl A, Khan IA, Ramakrishna W, San Miguel P, Bennetzen JL, Echenique V, Lijavetzky D, Dubcovsky J. The wheat $V R N-2$ gene is a flowering repressor down-regulated by vernalization. Science. 2004;303:1640-4.

14. Beales J, Turner A, Griffiths S, Snape JW, Laurie DA. A pseudo-response regulator is misexpressed in the photoperiod insensitive Ppd-D1amutant of wheat (Triticum aestivum L.). Theor Appl Genet. 2007;115:721-33.

15. Turner A, Beales J, Faure S, Dunford RP, Laurie DA. The pseudo-response regulator Ppd-H1 provides adaptation to photoperiod in barley. Science. 2005:310:1031-4.

16. Valárik M, Linkiewicz A, Dubcovsky J. A microcolinearity study at the earliness per se gene Eps-A $m 1$ region reveals an ancient duplication that preceded the wheat-rice divergence. Theor Appl Genet. 2006;112:945-57.

17. Pugsley AT. A genetic analysis of the spring-winter habit of growth in wheat. Aust J Agric Res. 1971;22:21-31.

18. Yan L, Helguera M, Kato K, Fukuyama S, Sherman J, Dubcovsky J. Allelic variation at the $\mathrm{VRN}-1$ promoter region in polyploidy wheat. Theor Appl Genet. 2004;109:1677-86.

19. Pidal B, Yan L, Fu D, Zhang F, Tranquilli G, Dubcoveky J. The CArG-box located upstream from the transcriptional start of wheat vernalization gene VRN1 is not necessary for the vernalization response. J Hered. 2009;100:355-64.

20. Golovnina KA, Kondratenko EY, Blinov AG, Goncharov NP. Molecular characterization of vernalization loci VRN1 in wild and cultivated wheats. BMC Plant Biol. 2010;10:168-82.

21. Shcherban AB, Strygina KV, Salina EA. VRN-1 gene- associated prerequisites of spring growth habit in wild tetraploid wheat $T$. dicoccoides and the diploid a genome species. BMC Plant Biol. 2015;15:94.

22. Yu M, Carver BF, Yan L. TamiR1123 originated from a family of miniature inverted-repeat transposable elements (MITE) including one inserted in the Vrn-A1a promoter in wheat. Plant Sci. 2014;215-216:117-23.

23. Li G, Yu M, Fang T, Cao S, Carver BF, Yan L. Vernalization requirement duration in winter wheat is controlled by TaVRN-A1 at the protein level. Plant J. 2013;76:742-53.

24. Fu DL, Szücs P, Yan LL, Helguera M, Skinner JS, Zitzewitz JV, Hayes PM, Dubcovsky J. Large deletions within the first intron in VRN-1 are associated with spring growth habit in barley and wheat. Mol Gen Genomics. 2005;273:54-65.

25. Chu CG, Tan CT, Yu GT, Xu SS, Yan L. A novel retrotransposon inserted in the dominant $V r n-B 1$ allele confers spring growth habit in tetraploid wheat (Triticum turgidum L.). Genes Genome Genet. 2011;1:637-45.

26. Bonnin I, Rousset M, Madur D, Sourdille P, Dupuits C, Brunel D, Goldringer I. FT genome $\mathrm{a}$ and $\mathrm{D}$ polymorphisms are associated with the variation of earliness components in hexaploid wheat. Theor Appl Genet. 2008;116:383-94.

27. Wang S, Carver BF, Yan L. Genetic loci in the photoperiod pathway interactively modulate reproductive development of winter wheat. Theor Appl Genet. 2009:118:1339-49.

28. Chen Y, Carver B, Wang S, Cao S, Yan L. Genetic regulation of developmental phases in winter wheat. Mol Breed. 2010:26:573-82.

29. Zhu X, Tan CT, Yan L. Molecular identification of null alleles at VRN-2 genes on a, B, and D genomes of hexaploid wheat. Mol Breed. 2011;27:501-10.

30. Tan CT, Yan L. Duplicated, deleted and translocated VRN2 genes in hexaploid wheat. Euphytica. 2016;208:277-84.

31. Iqbal M, Navabi A, Yang RC, Salmon DF, Spaner D. Molecular characterization of vernalization response genes in Canadian spring wheat. Genome. 2007:50:511-6

32. Shitsukawa N, Ikari C, Shimada S, Kitagawa S, Sakamoto K, Saito H, Ryuto $H_{\text {, }}$ Fukunishi N, Abe T, Takumi S, Nasuda S, Murai K. The einkorn wheat (Triticum monococcum) mutant, maintained vegetative phase, is caused by a deletion in the VRN-1 gene. Genes Genet Syst. 2007:82:167-70.

33. Loukoianov A, Yan L, Blechl A, Sanchez A, Dubcovsky J. Regulation of VRN-1 vernalization genes in normal and transgenic polyploid wheat. Plant Physiol. 2005;138:2364-73.

34. Zhang XK, Xia XC, Xiao YG, Zhang Y, He ZH. Allelic variation at the vernalization genes $V r n-A 1, V r n-B 1, V r n-D 1$ and $V r n-B 3$ in Chinese common wheat cultivars and their association with growth habit. Crop Sci. 2008;48:458-70.

35. Dubcovsky J, Loukoianov A, Fu D, Valarik M, Sanchez A, Yan L. Effect of photoperiod on the regulation of wheat vernalization genes $V R N-1$ and VRN-2. Plant Mol Biol. 2006;60:469-80.
36. Fowler DB, Breton G, Limin AE, Mahfoozi S, Sarhan F. Photoperiod and temperature interactions regulate low-temperature-induced gene expression in barley. Plant Physiol. 2001;127:1676-81.

37. Dyck JA, Matus-Cádiz MA, Hucl P, Talbert L, Hunt T, Dubuc JP, Nass H, Clayton G, Dobb J, Quick J. Agronomic performance of hard red spring wheat isolines sensitive and insensitive to photoperiod. Crop Sci. 2004:44:1976-81.

38. Kane NA, Danyluk J, Tardif G, Ouellet F, Laliberte JF, Limin AE, Fowler DB, Sarhan F. TaVRT-2, a member of the StMADS-11 clade of flowering repressors, is regulated by vernalization and photoperiod in wheat. Plant Physiol. 2005;138:2354-63.

39. Hemming MN, Peacock WJ, Dennis ES, Trevaskis B. Low-temperature and daylength cues are integrated to regulate FLOWERING LOCUS T in barley. Plant Physiol. 2008;147:355-66.

40. Chen Y, Carver BF, Wang S, Zhang F, Yan L. Genetic loci associated with stem elongation and winter dormancy release in wheat. Theor Appl Genet. 2009;118:881-9.

41. Dubcovsky J, Galvez AF, Dvorak J. Comparison of the genetic organization of the early salt stress response gene system in salt-tolerant Lophopyrum elongatum and salt-sensitive wheat. Theor Appl Genet. 1994;87:957-64.

42. Holland JB. EPISTACY: A SAS program for detecting two-locus epistatic interactions using genetic marker information. J Hered. 1998;89:374-5.

\section{Submit your next manuscript to BioMed Central and we will help you at every step:}

- We accept pre-submission inquiries

- Our selector tool helps you to find the most relevant journal

- We provide round the clock customer support

- Convenient online submission

- Thorough peer review

- Inclusion in PubMed and all major indexing services

- Maximum visibility for your research

Submit your manuscript at www.biomedcentral.com/submit
Biomed Central 